\title{
Prevalence of polycystic ovarian disease among students of tertiary care hospital of Kutch, Gujarat- a cross-sectional study
}

\author{
Vaghela S. ${ }^{1}$, Kotak P. ${ }^{2}$ \\ ${ }^{1}$ Dr. Shaktisinh Vaghela, ${ }^{2}$ Dr. Prafulla Kotak, both authors are Assistant Professor, Department of Obstetrics and \\ Gyanecology, Gujarat Adani Institute of Medical Science, Bhuj, Kutch, Gujarat, India \\ Corresponding Author: Dr Prafulla Kotak, Department of Obstetrics \& Gynecology, Gujarat Adani Institute of Medical \\ Science, Bhuj, Kutch, Gujarat, India. E-mail: researchguide86@gmail.com
}

\begin{abstract}
Background and Aim: Diagnosing Polycystic ovarian syndrome at an early stage will help prevent these complications of maternal and child health which appears to cover two of the eight objectives of millennium development goals (MDG). Present study was done with an aim to find its prevalence in medical, dental, nursing and paramedical female students, aged between 16-24 years, in a teaching hospital.Material and Methods:A cross sectional study was carried out among 112 subjects in a tertiary care teaching hospital of Kutch, Gujarat. Students of aged 16-25 years were randomly selected from various batches and branches of medical and paramedical courses. The diagnosis of PCOS was made based on Rotterdam's criteria. The ultrasound criteria define the polycystic ovary as containing 12 or more follicles measuring 2-9mm and/or an increased ovarian volume of $>10 \mathrm{~cm}$. Results: The average age of students with and without PCOS did not show much difference. The percent of students having only menstrual irregularity with cyst (14.79\%) which is higher than those having only the other two combinations $(6.1 \%$ and $1.6 \%)$ showing that a student with menstrual irregularity is more likely to have a cyst and vice versa.Conclusion:Based on the observation it is concluded that the prevalence of PCOS is increasing gradually in Areas of Kutch, Gujarat and it may be a major health concern in future. Hence, early diagnosis and intervention will reduce the long term health complications associated with PCOS.
\end{abstract}

Keywords: Kutch,Menstrual irregularity, Polycystic ovarian syndrome, Rotterdam's criteria

\section{Introduction}

Polycystic ovarian syndrome (PCOS) is one of the most common reproductive endocrinological disorders with a broad spectrum of clinical manifestations affecting about $6-8 \%$ of women of reproductive years [1]. The European Society of Human Reproduction and Embryology/American Society for Reproductive Medicine criteria, often called Rotterdam, includes various phenotypes based on a combination of any two of the three findings of hyperandrogenism, menstrual irregularity, and polycystic ovaries on ultrasound [2]. There are many interacting factors which result in clinical and/or biochemical hyperandrogenism. The symptoms of PCOS emerge slowly and these features are coincident with the changes of normal pubertal development. So these subtle features may not be recogonised in an early stages and may account for the failure to identify the disorder in young girls.

Manuscript received: $17^{\text {th }}$ September 2019

Reviewed: $23^{\text {th }}$ September 2019

Author Corrected: $28^{\text {th }}$ September 2019

Accepted for Publication: $3^{\text {th }}$ October 2019
Women with PCOS are at an increased risk of obstructive sleep apnea, infertility, dysfunctional uterine bleeding, metabolic syndrome, type 2 diabetes, cardiovascular disease, depression and certain cancers 3-10].

Diagnosing PCOS at an early stage will help prevent these complications of maternal and child health which appears to cover two of the eight objectives of millennium development goals (MDG) [11]. Preventing measures like exercises, healthy diet, practicing meditation, calming exercise that reduce stress levels etc. play an important role in reducing the complications of PCOS. There is a rapid rise in the incidence of obesity in children which results in metabolic syndrome, dyslipidemia, insulin resistance and polycystic ovarian syndrome. The prevalence in Asian countries appears to be lower, with a reported prevalence of $2.4 \%$ in Chinal and $6.3 \%$ in Sri Lanka (Rotterdam criteria). ${ }^{5}$ Prevalence was found to be $6.8 \%$ in north Indian women [12]. 


\section{Original Research Article}

In view of the above cited causes and the fact that prevalence of this syndrome in our community remains unknown, we attempted to find its prevalence in medical, dental, nursing and paramedical female students, aged between 16-24 years, in a teaching hospital.

\section{Material and Methods}

A cross sectional study was carried out among 112 subjects ina tertiary care teaching hospital of Kutch, Gujarat. The Institutional Informed consent was taken from the study participants and Ethics Committee approval was obtained prior to the study.Students of aged 16-25 years were randomly selected from various batches and branches of medical and paramedical courses.Although they were students of one college in a particular area, they represented a larger geographical area because they were from urban, semi-urban and rural areas around the district.

The study subjects were then distributed questionnaires which included queries pertaining to the presence of hyper androgenic features and menstrual irregularities (amenorrhoea/ oligomenorrhoea) The Female students doing course in the college and who were willing to go for an ultrasound examination were included in the study.

\section{Results}

One hundred and twelve students met the criteria to be diagnosed as polycystic ovarian syndrome. The demographic profile and the average age of onset of menstruation among the students are depicted in table 1. The percentage of students who met the Rotterdam's criteria is shown in table 2. Total number of students previously diagnosed with PCOS was $11.25 \%$. 6.1\% students had a history of thyroid dysfunction. Number of students, among those who have thyroid (as denominator); who also have now been diagnosed with PCOS was 59.9\%.

The other clinical features of hyperandrogenism among those who had PCOS were like oily skin (14.20\%), acne (9.01\%), increased hair growth (5.20\%), male pattern thinning of hair (8.90\%) and high degree of intolerance to cold and heat $(4.02 \%)$.

The other hyperandrogenic features were not considered in the diagnosis of PCOS as there is no standardized method (like Ferriman Gallwey method for hirsutism) that uses these features to satisfy the Rotterdam criteria of clinical hyperandrogenism. None of the students gave a self or family history of late onset congenital adrenal hyperplasia or galactorrhoea.

\section{Table-1: Demographic data of PCOS students}

\begin{tabular}{|l|c|c|}
\hline Variable & Subjects with PCOS & Subjects without PCOS \\
\hline Age & $17.80 \pm 2.14$ & $18.05 \pm 2.65$ \\
\hline BMI & $24.25 \pm 2.99$ & $23.31 \pm 4.02$ \\
\hline Average age if onset ofmenstruation & $8.15 \pm 0.78$ & $4.14 \pm 0.65$ \\
\hline mFG score & $13.06 \pm 0.76$ & $14.08 \pm 0.27$ \\
\hline
\end{tabular}

Obsgyne Review: Journal of Obstetrics and Gynecology

Available online at: www.medresearch.in 187 |P a g e 
Original Research Article

Table-2: Distribution of Patients according toRotterdam's criteria

\begin{tabular}{|l|c|}
\hline Clinical Feature & Percentage (\%) \\
\hline Irregular periods & 27.15 \\
\hline Cyst or increased stroma in ovaries & $32.25 \%$ \\
\hline Hirsutism (mFG) & $23.46 \%$ \\
\hline Both menstrual irregularity and cyst in ovaries & $22.06 \%$ \\
\hline Both cyst in ovaries and hirsutism & $14.79 \%$ \\
\hline Both menstrual irregularity and hirsutism & $10.01 \%$ \\
\hline Menstrual irregularity, cyst in ovaries and hirsutism & $7.9 \%$ \\
\hline
\end{tabular}

\section{Discussion}

PCOS among adolescents is an emerging problem that needs careful assessment, timely intervention, and appropriate treatment [13]. Thediversemanifestations of PCOS start at an early age when a girl is maturing into a young woman. During this pubertal transition, several features may be in evolution and thus many findings may be transitory which stabilize later during adolescence. However, it is important to make an early diagnosis in order to prevent early and late sequel of the syndrome.

PCOS is one of the most shared endocrine disorders among women but the diagnosis, management and the complications have not been consistent. Hyperandrogenism and menstrual irregularity are the most predominant symptoms of PCOS patients, but these symptoms are not absolute necessary for diagnosis of PCOS. Similarly, the ultrasonography indication of polycystic ovaries as a diagnostic marker doesn't validate much.

The average age of students with and without PCOS did not show much difference. Therefore there appears to be no difference in the incidence of PCOS in the various age groups between 16-25 years. In study conducted on Saudi girls, the estimated prevalence of PCOS was observed to be $53.7 \%$ which is strikingly higher. One of the explanations may be high incidence of obesity in Saudi Arabia which has an established association with PCOS[14]. Findings of the present study are also consistent with various studies done by Esmaeilzadeh $\mathrm{S}$ et al and Carmina E et al $[15,16]$.

PCOS is one of the most common endocrine disorders among women but the diagnosis, management and the complications have not been standardized. The increased prevalence of PCOS among young aged females may be due to unhealthy dietary habit like junk foods, lack of exercises, sedentary lifestyles. The childhood obesity can lead to insulin resistance and metabolic syndrome in later part of the life.
Stress is one of the major factors for all types' diseases now a day [17]. The changed lifestyle disorder can cause increased stress among the adolescents. So, the students should be stimulated to follow stress relieving techniques like yoga, meditations and to adapt the lifestyles. Studies done on familial PCOS suggested that subjects with classic features of PCOS, anovulation and hyperandrogenism, may have an affected sister who is equally hyperandrogenemic, but has regular cycles $[18,19]$ and polycystic ovaries [20].

The average BMI of those with PCOS is slightly higher than those without it. The average $\mathrm{mFG}$ scoring shows an average of 4.01 score increase in those with PCOS compared to those without. Among the three Rotterdam criteria the most common one is presence of follicles or increased stroma in the ovaries indicating that this is a common occurrence. Community based studies using Rotterdam criteria among reproductive age group women have demonstrated varied prevalence figures in few Asian countries ranging from $2 \%$ to $7.5 \%$ in China to $6.3 \%$ in Srilanka [21].

The percent of students having only menstrual irregularity with cyst $(14.79 \%)$ which is higher than those having only the other two combinations $(6.1 \%$ and $1.6 \%$ ) showing that a student with menstrual irregularity is more likely to have a cyst and vice versa. About $11.25 \%$ of the students had already been diagnosed with PCOS but the prevalence found was $31.20 \%$ indicating that there is a need for screening and early diagnosis and increased awareness about the condition.

About $59.9 \%$ of students with thyroid disorders also presented with PCOS and therefore there is a greater than half chance that adolescent girls might have PCOS if they are diagnosed with thyroid dysfunction. Similar reports were observed in a survey on 728 women wherein the prevalence was $8.7 \%$ according to $\mathrm{NIH}$ criteria and $17.8 \%$ according to Rotterdam criteria [22]. 


\section{Original Research Article}

The mFG score is a subjective one and in this study; the subject, the student undertaking the study and the guide have crosschecked for the grading of hirsutism thus reducing the subjectivity to an extent.

As this was cross-sectional study with limited sample size results cannot be generalized to the whole country population. This study can thus be followed up by a cohort study wherein we could monitor these students in the long run to observe and find out how many of them do develop complications in the future.

Thus it can be determined whether a diagnosis of PCOS at 16-25 years can actually predict the occurrence of complications and if so, to what extent.

\section{Conclusion}

Based on the observation it is concluded that the prevalence of PCOS is increasing gradually inAreas of Kutch, Gujarat and it may be a major health concern in future. Hence, early diagnosis and intervention willreduce the long term health complications associated with PCOS.

Addition of the study to exiting knowledge: The feasibility of conducting such communitybased study justifies the need to upscale this effort to get an overall estimate of the disorder in a diverse sociocultural and economic background, providing an opportunity for early detection and prevention of morbidities among adolescents and young women in India.

\section{Contribution from authors}

- Dr Shaktisinh Vaghela formulated the aims \& objectives with study design and helped in data collection from medical record department.

- Dr Prafulla Kotak,contributed to the preparation of the manuscript and Data analysis.

Sources of funding: Nil.

Conflict of interest: None declared

\section{References}

1. Azziz R, Marin C, Hoq L, Badamgarav E, Song P. Health care-related economic burden of the polycystic ovary syndrome during the reproductive life span. J ClinEndocrinolMetab2005; 90:4650-8.

2. Eshre R. Revised 2003 consensus on diagnostic criteria and longterm health risks related to polycystic ovary syndrome. Fertility and Sterility 2004;81(1):19.
3. Tasali E, Van Cauter E, Ehrmann DA. Relationships between sleep disordered breathing and glucose metabolism in polycystic ovary syndrome. J Clin Endocrinol Metab. 2006;91(1):36-42.

4. Giudice LC. Endometrium in PCOS: Implantation and predisposition to endocrine CA. Best Pract Res Clin Endocrinol Metab. 2006;20(2):235-44.

5. Cerda C, Perez-Ayuso RM, Riquelme A, Soza A, Villaseca P, Sir-Petermann T. Nonalcoholic fatty liver disease in women with polycystic ovary syndrome. J Hepatol. 2007;47(3):412-7.

6. Hollinrake E, Abreu A, Maifeld M, Van Voorhis BJ, Dokras A. Increased risk of depressive disorders in women with polycystic ovary syndrome. FertilSteril. 2007;87(6):1369-76.

7. RituDeswal, AmitaSuneja Dang. Prevalence of Poly Cystic Ovary Syndrome (PCOS) in North Indian Women - Indian journal of health and well-being. 2014;5(06):742-744.

8. Ram Nidhi, VenkatramPadmalatha, Raghuram Nagarathna. Ram Amritanshu. Prevalence of Polycystic Ovarian Syndrome in Indian Adolescents.

9. "List of goals, targets, and indicators". Available from www.worldbank.org. Retrieved. 2012 10-18.

10. Kalra S, Unni Krishnan A G. Obesity in India: The weight of the nation. J Med NutrNutraceut. 2012; 1:3741.

11. List of goals, targets, and indicators. Available at: www.worldbank.org.

12. Norman RJ, Dewailly D, Legro RS, Hickey TE. Polycystic ovary syndrome. Lancet. 2007; 370:685-97.

13. Gainie MA, Khurana ML, Eunice M, Gupta N, Diwivedi SN, Gulati MS, et al. Prevalence of glucose intolerance among adolescent and young women with polycystic ovary syndrome in India. Indian J EndocrinolMetab 2004.

14. Shaista Salman Guraya, Prevalence and ultrasound features of Polycystic ovaries in young unmarried Saudi females, Jounal of Microscopy and Ultrastructure:1(2013) 30-34. 


\section{Original Research Article}

15. Esmaeilzadeh S, Delavar MA, Amiri M, Khafri S, Pasha NG. Polycystic ovary syndrome in Iranian adolescents. Int J Adolesc Med Health. 2014;26(4):55965.

16. Carmina E, Lobo RA. Polycystic ovaries in hirsute women with normal menses. 2001;111(8):602-6.

17. Bindu BR. Increasing incidence of PCOS in adolescence and its relation with mental Stress. Res J Recent Sci. 2014;3(ISC-2013);227-30.

18. Enrico Carmina, Rogerio A Lobo. Polycystic ovaries in hirsute women with normal menses. 2001;111(8):602-606.

19. Carey, A.H., Chan, K.L., Short, F. Evidence for a single gene effect causing polycystic ovaries and male pattern baldness. Clin Endocrinol (Oxf). 1993;38(6):653-8.

20. Legro RS, Driscoll D, Strauss JF 3rd, Fox J, Dunaif A. Evidence for a genetic basis for hyperandrogenemia in polycystic ovary syndrome. Proc Natl Acad Sci U S A. 1998;95(25):14956-60.

21. Kumarapeli V, Seneviratne R de A, Wijeyaratne CN, Yapa RM., Dodampahala SH. A simple screeningapproach for assessing community prevalence and phenotypes of polycystic ovary syndrome in semiurban population in Srilanka. Am J Epidemiol 2008; 168:321-7.

22. March, A.W., Moore, V.M., Willson, K.J. The prevalence of polycystic ovary syndrome in a community sample assessed under contrasting diagnostic criteria. Hum Reprod. 2009; 25:544.

\section{How to cite this article?}

Vaghela S., Kotak P. Prevalence of polycystic ovarian disease among students of tertiary care hospital of Kutch, Gujarata cross-sectional study. Obgs Rev: J obstetGynecol 2019;5(4):186-190.doi:10.17511/joog.2019.104.02 